\title{
DIAGNÓSTICO DE LAS CONDICIONES DE SALUD DE LOS CARPINTEROS DEL QUINDÍO (COLOMBIA)
}

\author{
DIAGNOSIS OF HEALTH CONDITIONS OF THE CARPENTERS OF QUINDIO (COLOMBIA)
}

\author{
Milena Elizabeth. Gómez-Yepes ${ }^{1}$, Juan Farid Sánchez-López ${ }^{1}$, Paola Torres ${ }^{1}$, Deisy Sánchez ${ }^{1}$, Lina María Camargo ${ }^{1}$, Rene Arturo Castañeda ${ }^{1}$, \\ Jaime Fierro Pioquinto ${ }^{1}$, Angela María Cardona ${ }^{1}$, Rafael Humberto Villamizar ${ }^{1}$, Lazaro Vicente Cremades ${ }^{2}$. \\ ${ }^{1}$ Universidad del Quindío, Facultad Ciencias de la Salud, Programa de Salud Ocupacional. Armenia-Colombia. \\ ${ }^{2}$ Universitat Politécnica de Catalunya (UPC) - Barcelona TECH, Departamento de Proyectos de Ingeniería, ETSEIB, Barcelona, España.
}

Fecha de recibido: Febrero 3 de 2010

Fecha de aceptado: Junio 9 de 2010

Correspondencia: programa de salud ocupacional, Universidad del Quindío. Av. Bolivar calle 12 norte Armenia Quindío. Correo electrónico:

\section{RESUMEN}

Se realizó un estudio descriptivo observacional de las condiciones de salud de los trabajadores de las áreas de lijado, cepillado y pinturas (trabajadores con mayor probabilidad de sufrir problemas o enfermedades respiratorias por exposición al polvo de madera). Teniendo en cuenta la similitud de tareas, utilización de materiales y equipos se tomó una muestra por conveniencia de 10 empresas más representativas del Quindío. Se encuestaron el 100\% de los trabajadores de las áreas antes mencionadas. El 22.6\% dieron positivo a problemas respiratorios; estos trabajadores fueron elegidos para la elaboración de las historias clínicas ocupacionales. Se detectó el uso de maderas duras y blandas en la elaboración del mueble, así como inmunizantes o plaguicidas, solventes orgánicos volátiles, resinas epóxicas sintéticas, pegamentos y pinturas para los acabados de la madera. Del total de los encuestados, $45 \%$ eran mujeres y $55 \%$ hombres, siendo las mujeres cabeza de hogar. El promedio de edad fue de 31 años, el más joven de 19 y el mayor de 51 años. Con respecto a los hábitos, el 20\% son fumadores y el 45\% ingieren alcohol. El 45\% practican deportes y el 100\% negó padecer alguna enfermedad profesional. El 100\% de los trabajadores se quejó de de sufrir de tos, ronquera, renitis, conjuntivitis y gripas frecuentes. Es notable la falta de estilos de vida saludables y de programas de vigilancia epidemiológica de las empresas, que, junto a las condiciones ambientales existentes en sus áreas de trabajo, generan un importante factor de riesgo para eventos crónicos de salud, como la enfermedad pulmonar obstructiva crónica(EPOC).

Palabras clave: Condiciones de salud, carpinteros, polvo de madera, EPOC.

\section{ABSTRACT}

A descriptive analysis was carried out about health conditions of workers exposed to wood dust in the areas of sanding, planing and painting (this kind of workers are most likely to suffer respiratory problems or illness from exposure to wood dust). Given the similarity of tasks, materials and equipment used, we took ten of the representative companies in Quindio as the control group. The study surveyed $100 \%$ of workers in the above areas. $22.6 \%$ tested positive for respiratory problems, and these workers were chosen for the development of occupational medical records. We identified the use of hardwoods and softwoods in furniture making; immunization agents or pesticides; volatile organic solvents; synthetic epoxy resins; adhesives and paints for wood finishes. Of the total respondents, 45\% were women (all of whom where heads of households), and 55\% men. The average age was 31 years, with the youngest 19 years, and the eldest 51 years old. With regard to personal health habits, 20\% are smokers and $45 \%$ drink alcohol. $45 \%$ play sports and $100 \%$ denied suffering any occupational disease. $100 \%$ of workers complained of suffering from coughs, hoarseness, rhinitis, conjunctivitis and frequent colds. A notable lack of healthy lifestyles and epidemiological surveillance company programs (together with the environmental conditions in their areas of work) generate a significant risk factor for chronic health events, such as obstructive lung disease (COPD).

Key words: Health conditions, carpenters, wood dust, COPD.

Rev. Invest. Univ. Quindío (20): 171 - 178. Armenia - Colombia 


\section{INTRODUCCIÓN}

Una de las principales actividades económicas del Departamento del Quindío es el sector maderero desde el cultivo de árboles hasta su transformación en muebles. En el año 2004, en el municipio de Armenia se registraron aproximadamente 170 empresas madereras, dedicadas a la fabricación de muebles, ventas de productos terminados y de subproductos, siendo en su gran mayoría manufactura artesanal y casera informal (1).

La manufactura de la madera involucra procesos y operaciones relacionados principalmente con la tecnificación, lo que aumenta la probabilidad de ocurrencia de eventos ocupacionales. Además, se le mezclan a la madera sustancias químicas peligrosas para el acabado, lo que incrementa el riesgo de enfermedad y muerte del trabajador más rapidamente, empeorando así su calidad de vida laboral (2). Estos procesos de tecnificiación involucran riesgos higienicos como maquinaria con ruidos superiores a los $85 \mathrm{~dB}(\mathrm{~A})$, material particulado (polvo de madera), aspersión de solventes orgánicos y pinturas, entre otras (39).

El polvo de madera es una mezcla compleja de celulosa, fundamentalmente de poliosas y lignina. Las maderas utilizadas en la fabricación de muebles pueden ser duras y blandas. Las maderas duras tienen una mayor densidad $\left(\mathrm{g} / \mathrm{cm}^{3}\right)$ que las maderas blandas, fibras más cortas y con un mayor contenido de poliosas, quemicelulosas y sustancias solubles en solventes polares (taninos, flavonoides, quinonas y menor contenido de lignina que las maderas blandas). Las maderas duras son aquellas que proceden de árboles de hojas cadúceas que tardan décadas e inclusos siglos en alcanzar el grado de madurez suficiente para ser cortado y poder ser implicada en la elaboración de muebles o vigas de caseríos o viviendas unifamiliares, son más costosos y atractivos que las maderas blandas y son más apetecidas (10). Las maderas y sus subproductos en general son irritantes, alergenos y cancerígenos. Las maderas duras son cancerígenas, pues se tiene información sobre la aparición de cáncer en la cavidad nasal y senos paranasales asociado con la exposición de polvo de madera (2, 9, 11-15). Las maderas blandas pueden llegar a generar problemas alérgicos y de sensibilización en los trabajadores, como sinusitis, asma ocupacional, enfermedades respiratorias infecciosas a largo plazo, irritación de vías respiratorias, renitis y bronquitis crónica (7-8, 16-20). Igualmente pueden producir en piel eczemas y urticaria (17). Las maderas irritantes como el cocobolo, el ébano y jacaranda, pueden producir dermatitis o irritación en la piel, maderas reconocidas como alergenos son los abedules, las pinacéas, caobas, arrayanes, cornáceas, o moreras. Y si a estos procesos de transformación se le añade el uso de solventes organicos, pinturas e inmunizantes, se acrecienta el riesgo de enfermedades respiratorias y neurológicas (21-26). Los inmunizantes o plaguicidas utilizados para la conservación de la madera al ataque de plagas son liposolubles, lo que permite su acumulación en ambientes hidrofóbicos, tales como la materia orgánica del suelo, las plantas y los animales. Dichas sustancias químicas orgánicas los convierte en neurotóxicos potenciales en razón de la riqueza grasa del sistema nervioso (25). Los más utilizados son de tipo organofosforado y organoclorados como DEF, DFP, EPN, Haloxón, Leptophos, Merphos, Mipafox, Parathion, Tricloronate (Phytosol), Triclorfón (Dipterex, Cebirán), Tamarón, (Methamidolphos), Tri-orto-Cresil -Fosfato (TOCF), Malathión, Dimethoate, Monocrotophos, Fenthion, Dursban y Diazinon (25).

Puesto que, adicionalmente, se utilizan inmunizantes, solventes organicos volatiles, resinas epóxicas sintéticas, pegamentes, pinturas, entre otros en la transformación de la madera, aumenta el riesgo de adquirir enfermedades neurológicas y daños en el sistema nervioso central (27). Desde el punto de vista toxicológico, se debe tener en cuenta que los plaguicidas, además del compuesto químico que combate la plaga, contiene sustancias en las cuales se disuelve el producto, generalmente solventes orgánicos, cuyas propiedades tóxicas pueden ser mayores que las del mismo principio activo (25). Dentro de los plaguicidas, los llamados organoclorados parecen alterar la estructura mielínica de las fibras nerviosas provocando transtornos de la conducción nerviosa. Los plaguicidas organofosforados actúan sobre el sistema nervioso central a partir de la inhibición de la colinesterasa, sustancia básica en el proceso electroquímico de transmisión de los impulsos nerviosos. (25-28).

Desde el punto de vista del conocimiento, la realización de esta investigación permite ahondar en las características reales de las condiciones de trabajo, particularmente del polvo de madera del sector de las carpinterías del Quindío. Con ello se pretende tener un mayor sustento a la hora de tomar las medidas que permitan mejorar sustancialmente las condiciones de vida de los trabajadores del sector gracias a la prevención de la salud ocupacional (29).

\section{Material y métodos}

Tipo de estudio: Se realizó un estudio descriptivo, observacional de las condiciones de salud de los trabajadores expuestos a riesgos químicos de las carpinterías del Quindío. El análisis descriptivo permite superar la etapa de la observación y la recolección de datos, envuelve operaciones analíticas de la formación de 
evidencias empíricas representativas dentro del «proceso de reconstrucción de la realidad del objeto» (30), a través de "métodos técnicos", claramente definidos. La descripción puede utilizar vocabulario ordinario y simbólico para explicar los fenómenos y las interpretaciones que se hacen de la realidad. La descripción como base de las interpretaciones abstractas de los datos, y de la construcción de teorías, es un medio para explicar las manifestaciones de los informantes u observaciones y extender las conceptualizaciones (31). El carácter observacional dentro del proceso investigativo le permite a los estudiantes investigadores cierto nivel de inserción entre los informantes y por consiguiente entrar en contacto con la realidad investigada, a través de instrumentos claramente definidos para avanzar en la recolección de datos que luego servirán de base para el análisis de la información.

Población y Muestra: Dado que las condiciones del ambiente de trabajo de este sector son similares, en cuanto a los equipos de trabajo, al igual que las materias primas y los insumos empleados, se ha optado en razón de la limitación presupuestaria, por una muestra por conveniencia (32-34), motivo por el cual se seleccionaron 10 empresas del sector. Los instrumentos para la recolección de la información de las condiciones de salud, fueron diseñados de acuerdo a los parámetros del Ministerio de Protección Social de Colombia y la OMS $(14,35)$. El total de los trabajadores de las empresas seleccionadas fue de 650 y se escogieron el $100 \%$ de los trabajadores (177) de las áreas de lijado, cepillado, pintura e inmunizado, trabajadores con alto riesgo de adquirir problemas o enfermedades respiratorias por la exposición al polvo de madera $(32,34)$.

Autorreporte de la condiciones de salud: Las condiciones de salud son un conjunto de actividades tendentes a la evaluación de la morbimortalidad y absentismo laboral por eventos ocupacionales o ATEP generada por las condiciones de trabajo. Para la información recogida en las encuestas, primó el salvaguardar el secreto profesional y la confidencialidad de los datos obtenidos a través de los trabajadores, de acuerdo a lo establecido en las Normas Científicas y Administrativas para la Investigación en Salud, establecidas por la Resolución 08430 de 1993 del Ministerio de Salud de Colombia. En las encuestas no se incluyó el dato del nombre del trabajador, ni la dirección de su domicilio, y antes de diligenciar la encuesta, al trabajador se le notificó del consentimiento informado. A partir de las 177 encuestas realizadas a los trabajadores, el médico especialista en salud ocupacional seleccionó aquellas encuestas de morbilidad sentida con resultados positivos a las preguntas de problemas respiratorios que de alguna manera tenían que ver con los contaminantes químicos presentes en la fabricación de muebles para elaboración de las historias clínicas ocupacionales. Del total de los trabajadores encuestados se seleccionaron 40 , o sea, el $22.5 \%$ de los trabajadores encuestados.

Análisis estadísticos: para las variables relacionadas con personas, se ha utilizado la estadística descriptiva: medidas de tendencia central y dispersión: rango, media, mediana, moda, desviación estándar, proporciones o porcentajes (36). Para resumir los datos se han empleado gráficos dependiendo del tipo de variables: análisis de la accidentalidad laboral, índice de frecuencia, índice de severidad y el índice de lesiones incapacitantes.

\section{Resultados}

Insumos utilizados en las fábricas visitadas: Tipos de madera utilizados en las fábricas: Se detectó el uso de maderas duras, como el Cedro Negro, Cedro Tolua, Nogal, Cedro Rosado, Cedro Caracol, Cedro Gino y maderas blandas como Chapul, Karra No. 1, Karra No. 2, Laurel mora, Nazareno, Perillo antioqueño, Pino canadiense, Pino romero, romero liso, Sajo, Vakera, Tablex corriente de 12 $\mathrm{mm}$, Tablex enchapado de $12 \mathrm{~mm}$, Triples de $4 \mathrm{~mm}$, MDF $4 \mathrm{~mm}$, MDF de $15 \mathrm{~mm}$ enchapada, MDF de $15 \mathrm{~mm}$.

Tipos de acabado: se detectó el uso de resinas sintéticas como el sellador caramelo, sellador mocca, veta simulado, terminado caramelo, terminado mocca; pegantes tipo cola (base de polipropilenos), presellantes (resinas epóxicas), sellantes (base de polivinilos de acetato), adhesivos, alquitran, thinner, barsol, gasolina.

Tipos de inmunizantes y/o plaguicidas: Dursban (plaguicida organofosforado-organotiofosfato piridina), el piretroide Demon o Cipemetrina.

Autorreporte de las condiciones de salud: Las edades de los 177 trabajadores que respondieron el autorreporte de morbilidad sentida, se distribuyeron por grupos quinquenales dando como resultado que el $50 \%$ de los trabajadores tienen edades comprendidas entre 20 y 29 años. El trabajador más joven tiene 15 años y el mayor 65 (media: 28; desviación estándar: 9.8; moda: 24). Del total de los encuestados, $45 \%$ eran mujeres y $55 \%$ hombres, siendo las mujeres cabeza de hogar. El promedio de edad fue de 31 años, el más joven de 19 y el mayor de 51 años. En la Figura 1 se puede observar el género y la edad de los trabajadores encuestados. 


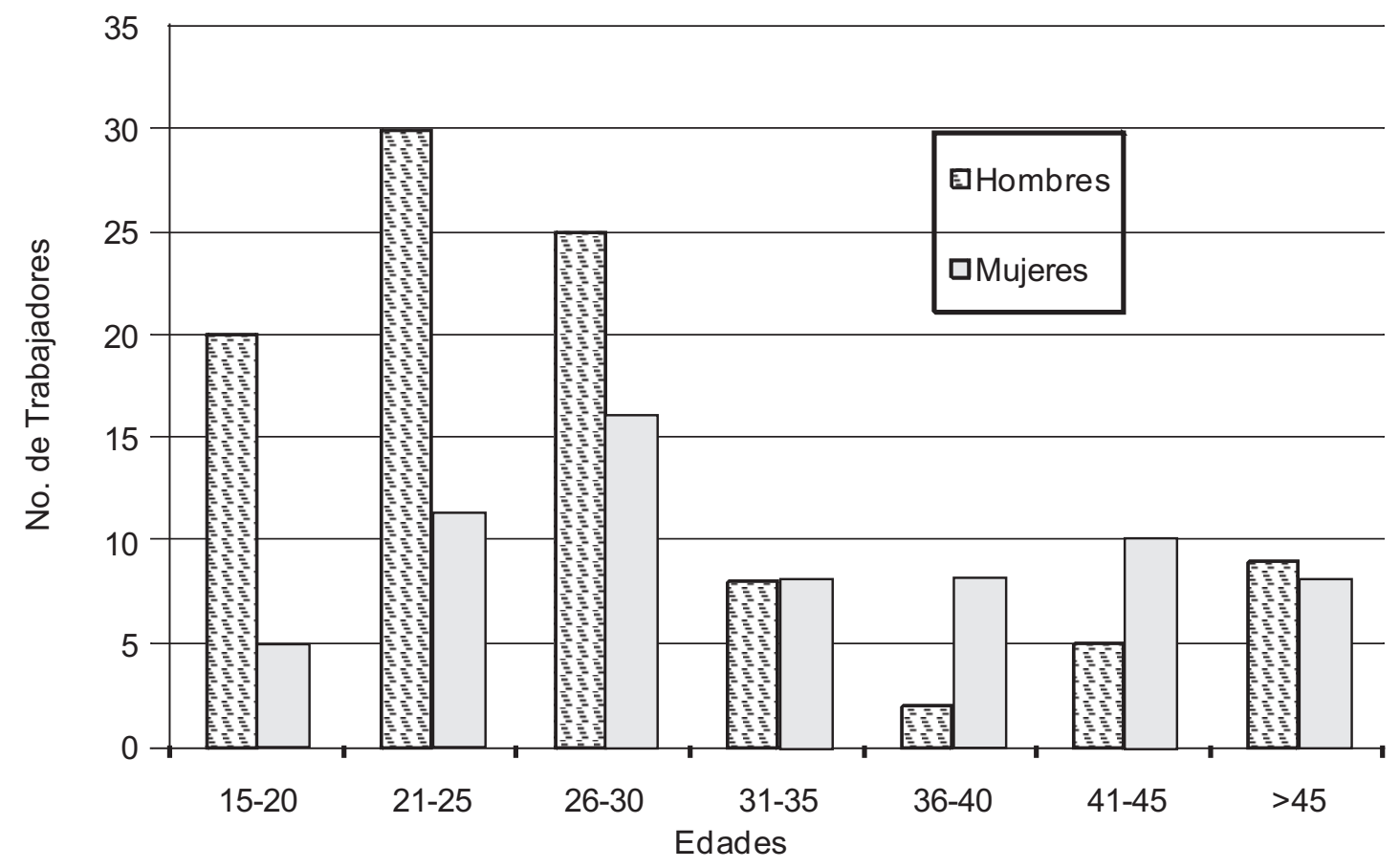

Figura 1. Género y edades de los trabajadores seleccionados y encuestados de las áreas de pulido, cepillado y pinturas, tomadas del autorreporte de morbilidad sentida hecho por los trabajadores.

Con respecto a las diferentes variables del autorreporte, se realizó una agrupación de aquellas que están representando unas inadecuadas condiciones en el ambiente de trabajo en el proceso de la fabricación de los muebles de madera, y son las siguientes:

- Ardor, irritación o enrojecimiento en los ojos: ésto fue referido por el $40.4 \%$ de los trabajadores, lo que nos da una idea de las condiciones de contaminación ambiental y/o de la falta de un adecuado uso de protección visual.

- Congestión nasal y/o frontal: referido por el $17.4 \%$ de los trabajadores, que puede estar dando luces con respecto a los eventos que afectan la vía respiratoria superior, incluyendo los eventos en los que puede participar la contaminación ambiental propia de este tipo de industrias.

- Secreción nasal, mal olor nasal: sólo fue referido por el $6.7 \%$ de los trabajadores, y hace referencia a la complicación de tipo infeccioso de un evento de la vía aérea superior.

- Ataques (accesos) de tos: indicado por el $14.6 \%$ del personal; desgarros, $10.6 \%$; ronquera, $14.6 \%$; carraspera en la garganta, $28.6 \%$.
- Falta de aire al subir escalas: el $21.9 \%$, aunque las fábricas visitadas en su inmensa mayoría son de una sola planta y el $50 \%$ de los trabajadores se encuentran en la segunda década de la vida. No se debe olvidar en este punto las condiciones de adaptación física.

- Sensación de opresión en el pecho: $11.8 \%$, al igual que otros síntomas referidos al esfuerzo.

- Falta de aire nocturno (cuando se está en cama): 3.3\%,y de ellos sólo dos trabajadores son mayores de cuarenta años. Aquí la subjetividad en la valoración del síntoma juega un papel muy importante.

- Ha tenido enfermedad profesional: un 3.9\%, aunque en los reportes revisados no aparece notificación alguna de este tipo de evento ocupacional.

- Alergia en la piel: $15.7 \%$, sin hacer en este punto una discriminación de si el evento de salud se ha iniciado en la empresa o fuera de ella.

- Resequedad en la piel: en un $26.4 \%$.

- Hongos en alguna parte del cuerpo: en un 9.5\%, principalmente asociados a condiciones de humedad en los pies. 
Historias clínicas ocupacionales de los trabajadores: A partir de las 177 encuestas realizadas a los trabajadores, el médico especialista en salud ocupacional seleccionó aquellas encuestas de morbilidad sentida con resultados positivos a las preguntas de problemas respiratorios que de alguna manera tenían que ver con los contaminantes químicos presentes en la fabricación de muebles para elaboración de las historias clínicas ocupacionales. Del total de los trabajadores encuestados se seleccionaron 40, o sea, el $22.5 \%$ de los trabajadores encuestados.

La percepción de las condiciones de salud en los trabajadores se valoró mediante un instrumento compuesto por 59 preguntas orientadas a síntomas dependientes de los diferentes sistemas orgánicos, con especial énfasis en aquellos que más se pueden comprometer por las tareas e insumos propios del quehacer de la industria del mueble.

Con respecto al estado civil (figura 2), llama la atención cómo la modalidad predominante es la unión libre, mostrando la estratificación socioeconómica de procedencia de los trabajadores de este tipo de empresas y sus preferencias por el tipo de vinculación marital, con todas las implicaciones que esto conlleva.

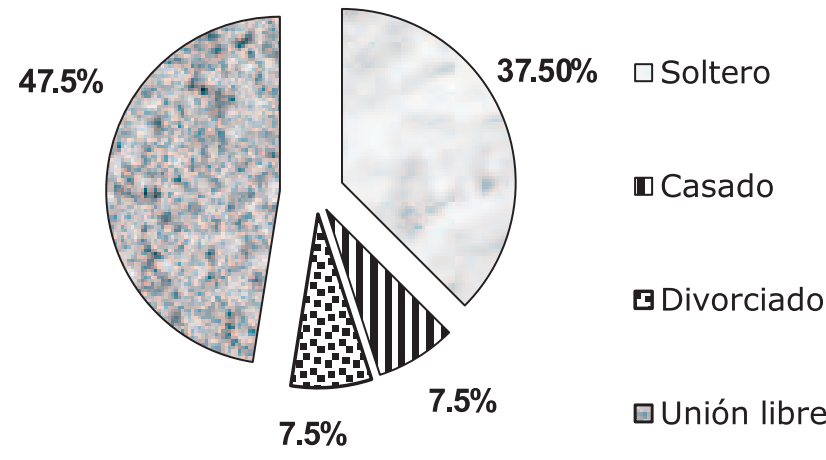

Figura 2. Estado civil de los trabajadores, registro tomado de las historias clínicas ocupacionales (HCO)

Del total de los trabajadores, el 17,5\% (7) no están afiliados a una Empresa Promotora de Salud (EPS), sino que cuentan con afiliación a una Administradora del Régimen Subsidiado (ARS). Con respecto a la afiliación a un fondo de pensiones, solamente el $25 \%$ (10) están afiliados y el 37,5\% (15) tienen Administradora de Riesgos Profesionales.

Edad: Del total de los trabajadores escogidos para las historias clínicas. Del total de los encuestados, $45 \%$ eran mujeres y $55 \%$ hombres, siendo las mujeres cabeza de hogar. El promedio de edad fue de 31 años, el más joven de 19 y el mayor de 51 años con una desviación estándar de 10.1 .
Antigüedad: Analizando la antig edad en la empresa, el $62,5 \%$ de los trabajadores están dentro del primer año de trabajo, muy pocos sobrepasan la barrera de los cinco años y en todos los casos se trata de empleados de confianza o de aquellos que se desempeñan en labores muy especializadas, y por lo tanto representan un gran valor para la empresa. Los cargos de los trabajadores a los que se les realizó la historia clínica son: $37,5 \%$, lijadores; el $15 \%$, operarios de maquinaria, y un $47,5 \%$ están en cepillado, inmunizado y pintura.

Hábitos: El 20\% de los examinados fuman y el $45 \%$ ingieren alcohol.

Ejercicio físico: El 45\% de los trabajadores refiere practicar algún tipo de actividad deportiva al menos una vez a la semana, manifestando además que su jornada de trabajo es la razón de no poder tener una mayor dedicación al deporte.

Eventos ocupacionales: Con respecto a la ocurrencia de accidentes de trabajo o enfermedades ocupacionales durante su vida laboral, encontramos que el $27,5 \%$ refieren haber tenido un accidente reconocido como tal y el $100 \%$ niegan tener una enfermedad profesional.

Revisión por sistemas: Interrogados los trabajadores, el $65 \%$ refiere que durante los últimos dos meses han presentado algún síntoma relacionado con diversos eventos de salud que si bien no han sido incapacitantes los han llevado a requerir consultar o a auto-formularse algún tipo de medicamento.

\section{Hallazgos en el interrogatorio importantes para su ocupación:}

- Empleo de aditamentos extraños (papel) dentro de los protectores respiratorios con la falsa idea de aumentar su eficacia.

- Inadecuada frecuencia de cambio de los filtros de los protectores respiratorios.

- No uso de los equipos de protección personal durante toda la jornada de trabajo.

- Algunos trabajadores manifestaron que los protectores respiratorios no son de una adecuada calidad para retener el polvo.

Como se puede observar en la Figura 3, el $26.5 \%$ de los trabajadores tiene problemas de salud visual, un $15 \%$ de varices en los miembros inferiores, un $15 \%$ de escoliosis y un $15 \%$ de faringitis. 


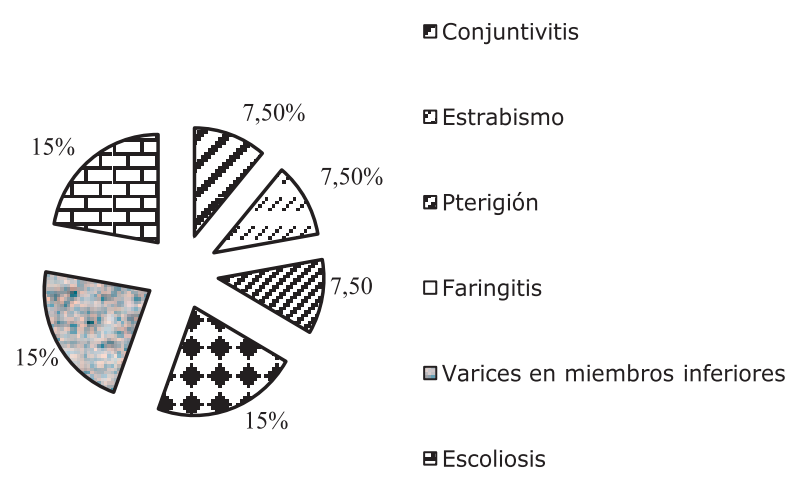

Figura 3. Resultados del examen físico realizado a los trabajadores seleccionados para la realización de las historias clínicas ocupacionales (Universidad del Quindío, 2006).

Análisis de absentismo e incapacidad laboral en los trabajadores del sector maderero: Respecto a la ocurrencia de eventos ocupacionales, encontramos que el $27,5 \%$ de los trabajadores encuestados refiere haber tenido un accidente reconocido como tal, y el $100 \%$ niega tener una enfermedad profesional. Se detectó que ninguna de las empresas lleva el programa de vigilancia epidemiológica de material particulado.

\section{Discusión}

Insumos utilizados en las fábricas visitadas: Durante las visitas de inspección a las fábricas se detectó el uso de maderas duras y maderas blancas, además de la utilización de pesticidas, solventes organicos volatiles, resinas sintéticas, pinturas y sellantes necesarios para el acabado de la madera. Es sabido que los riesgos de la exposición al polvo de maderas (duras y blandas) pueden llegar a generar problemas o enfermedades ocupacionales (asma ocupacional, bronquitis aguda, EPOC e incluso cáncer nasal y pulmonar). Además, la exposición continúa de los compuestos orgánicos volátiles y de plaguicidas organofosforados puede llegar a generar intoxicaciones crónicas neurológicas a los trabajadores expuestos.

Autorreporte de las condiciones de salud: Cabe señalar que un porcentaje importante de la población trabajadora (39.9\%) son mujeres cabeza de hogar, generalmente madres solteras, inician muy jóvenes las labores de carpinteria y permanecen más tiempo en el oficio. Los problemas de salud que más señalaron los trabajadores en las encuestas son el ardor, la irritación, congestión nasal, dolor de cabeza, tos, que pueden relacionarse con la exposición prolongada al polvo presente en el medio ambiente laboral. Los trabajadores mayores de 40 años manifestaron tener molestias respiratorias al subir escaleras y al realizar trabajos fuertes manifestaron de sufrir opresión en el pecho.

Manifestaron problemas de la piel, irritación y alergias que pueden estar asociadas con el tipo de elementos químicos que se emplean en el proceso de fabricación de los muebles de madera.

Historias clínicas ocupacionales (HCO): Un porcentaje inferior al $50 \%$ de los trabajadores no está cubierto por el régimen contributivo al Sistema General de Seguridad Social Integral (SGSS). Este punto es muy importante si tenemos en cuenta los riesgos tan relevantes a los cuales están expuestos los trabajadores de las empresas dedicadas a la fabricación de muebles de madera, por lo que presumimos un trabajo al destajo, de contratación informal y de una escasa vigilancia por parte de entidades del gobierno en el cumplimiento de aportes y protección del trabajador al SGSS; por lo tanto, se desconoce la existencia de enfermedades ocupacionales generadas por la exposición al polvo de madera (37-39).

Predominan los malos hábitos laborales de los trabajadores como el fumar e ingerir alcohol. En el primero de los hábitos influye el hecho de entenderse el efecto negativo que para la salud representa el consumo de tabaco, mientras que el consumo de alcohol está arraigado como un hecho social y su efecto nocivo para la salud no es comprendido tan fácilmente como el del tabaco.

Durante casi toda la jornada laboral (10 horas/día) se trabaja de pie, los trabajadores manifestaron problemas en las extremidades inferiores, problemas de espalda. Además en las HCO manifestaron irritación en los ojos y gargantas, razón que nos lleva a pensar la alta concentración del polvo de madera en el ambiente.

Análisis de absentismo e incapacidad laboral en los trabajadores del sector maderero: Los coordinadores de Salud Ocupacional y de Seguridad Industrial de las empresas manifestaron no presentar ningún tipo de absentismo con respecto a la exposición del polvo de madera. Sin embargo, son frecuentes los accidentes de trabajo relacionados con la manipulación de herramientas y maquinaria. Sin embargo, se considera un subregistro debido a que el personal entrevistado se quejó de sufrir de tos, ronquera, rinitis y gripes frecuentes. Es sorprendente la falta de seguimiento epidemiológico de los trabajadores, aunque lo define la Ley Colombiana como obligatorio; se aprecia por tanto una evasión de responsabilidades de los empleadores con la normatividad vigente en materia de seguridad social (37).

\section{Conclusiones}

En el análisis de las condiciones de salud se constató una notable falta de estilos de vida saludables de los trabajadores, ya que persisten hábitos con serias 
repercusiones en las condiciones de salud y en el trabajo, como la ingesta de bebidas alcohólicas y consumo del tabaco. Todo ello, unido a las condiciones ambientales existentes en sus áreas de trabajo, genera un importante factor de riesgo para eventos crónicos de salud, como la enfermedad pulmonar obstructiva crónica (EPOC). La escasa motivación existente en el grupo de trabajadores por mantener unas mínimas condiciones de seguridad, que aunque es un precepto constitucional (art. 49, Constitución Política de Colombia), requiere un importante grado de concienciación y sensibilización, expresado esto por la falta de elementos de protección personal, mantenimiento y cuidados. Además, la evasión de responsabilidades por parte de los empleadores en la implementación de programas de vigilancia epidemiológica, salud e higiene ocupacional, agrava la problemática respecto al trabajador como parte integral en el desarrollo sociocultural de la región.

\section{Trabajo futuro}

El estudio de las 10 empresas madereras del departamento del Quindío ha conllevado a formular proyectos de futuras investigaciones referentes al estudio de las condiciones de trabajo y salud de los carpinteros de la región del eje cafetero colombiano, como las siguientes:

- Elaboración de estrategias metodológicas de proyección social que permitan sensibilizar y concienciar a empleadores y trabajadores, con respecto al autocuidado de estilos de vida saludables (dicho proyecto se ejecutó en el 1으 semestre de 2007 Fuente: Programa de Salud Ocupacional, Universidad del Quindío).

- Implementación de un sistema de vigilancia epidemiológica para trabajadores expuestos a material particulado de la madera y poder detectar con más certeza las enfermedades respiratorias crónicas.

- Realizar un estudio de morbimortalidad laboral de los carpinteros y analizar el riesgo atribuible de absentismo por enfermedades o problemas respiratorios debido a la exposición al polvo de madera.

\section{BIBLIOGRAFÍA}

1. Cámara de Comercio de Armenia. Estadísticas Registros mercantiles. Armenia (Colombia). 2004.

2. Organización Internacional del Trabajo. XVI en el XVI Congreso Mundial sobre Seguridad y Salud en el Trabajo. Organización Internacional del Trabajo (OIT). Viena. 2002.

3. Casal J, Montiel H, Planas E, Vilchez J. Análisis del riesgo en instalaciones industriales. España. Ediciones UPC. 2001.

4. Chih-Shan L, Li-Yuan HSU. Elemental profiles of indoor and outdoor particulate matter less than 10 vans (pm10) and 2.5 $\tan$ (pm_2.5) in Taipei. Chemosphere. 1993; 27: (11) 2143-2154.

5. Friesen MC, Davies HW, Teschke K, Marion S, Demers PA. Predicting Historical dust and wood dust exposure in sawmills model development and validation, Journal of Occupational and Environmental Hygiene. 2005; 2(12):650.

6. Gómez-Yepes ME, Sánchez-López JF, Villamizar RH, Pioquinto JF, Torres P, Sánchez D, et. al. Health and working conditions in carpenter's workshops in Armenia (Colombia). Industrial Health. (accepted on July 30th, 2009, in press).

7. Manufactura Venezolana SA. Procesos de la Fabricación de la Madera. División de productos para la protección respiratoria, auditiva y ambiental de 3M. Caracas. 2002.

8. National Institute for Occupational Safety and Health. NIOSH-DOD-OSHA SPONSORED. Chemical and Biological Respiratory Protection Workshop Report. U.S. Department Of Health And Human Services Public Health Service. Centers for Disease Control and Prevention, National Institute for Occupational Safety and Health, February 2000; Publication No. 2000-122.

9. Occupational Safety and Health Administration. Wood dust. Comments from the January 19.Final Rule on Air Contaminants Project extracted from 54FR2332 ET. Seq. This rule was remanded by the U.S. Circuit Court of Appeals and the limits are not currently in force. USA. 1989.

10. Mutua Navarra. Ficha Técnica de higiene I. El polvo de Madera dura es carcinogeno. Dpto. de Prevención. Navarra. España. 2004

11. American Conference of Governmental Industrial Hygienists. TLV'S and BEL'S Thereshold Limit Values for Chemical Substances and Physcal agent. ACGIH. Wordwide. Cincinnati. Ohio. USA. 2008.

12. Magnavita N, Sacco A, Bevilacqua L, D'Alessandris T, Bosman C. Aesthesioneuroblastoma in a woodworker. Occupational Medicine. Health \& Medical Complete. 2003; 53(3) 231.

13. Organización Internacional del Trabajo. 91 a Sesión de la Conferencia Internacional del Trabajo (OIT). Ginebra. 2003 
14. Organización Mundial de la Salud OMS. Epidemiología de las enfermedades y accidentes relacionados con el trabajo. Décimo informe del Comité Mixto OIT/OMS sobre Higiene del Trabajo. ISBN 0509-2507. Ginebra. 1989.

15. Warhert DB, Hart GA, Hesterberg TW, Collins JJ, Dyer WM, Swaen GMH et al. Potential pulmonary effects of mans made organic fiber (MMOF) dust. Critical Reviews in toxicology Health\&Medical complete. 2001; 31(6): 697-736.

16. Asgharian B, Menache MG, Millar FJ. Modelling age-related particle deposition in humans. Journal of Aerosol Medicine. 2004; 17(3): 213-224.

17. Becerril J. Enfermedades de productos químicos. Revista Protección y Seguridad del Consejo Colombiano de Seguridad. 2004; Año 50 298: 24-34.

18. Cordoba D. Toxicología. 4a ed. Bogota. 2001

19. National Institute for Occupational Safety and Health.Control of wood dust from large diameter disc sanders. Appl. Occup. Environ Hygiene. 2001; 16(11):1022.

20. Sagrera JC, Mcdonald JD. Lung toxicity of ambient particulate matter from Souheasterns us: sites with different contributing sources. Relation ships between composition and effects Enviromental Health perspectives. Research Triangle Park. 2006; 114(9):1387.

21. Consejo Colombiano de Seguridad. Contaminantes químicos. Bogotá. Revista Salud, Trabajo y Ambientes. III Trimestre de 2003; 10 (37).

22. Espinosa MT. Neurotoxicidad asociada con exposición a sustancias químicas. Revista Salud, Trabajo y Ambiente del Consejo. Colombiano de Seguridad II Trimestre de 1995; 2 (4).

23. Londoño J. Higiene III. Factores de Riesgo Químico. Facultad de educación Abierta y a Distancia. Programa de Salud Ocupacional. Universidad del Quindío. Armenia (Colombia). 1996.

24. Manual de Higiene Industrial. Toxicología de los contaminantes químicos. Fundación MAPFRE. España. $1991 ;$ pp. 96 - 111.

25. Pradilla A. Neurotoxicidad por plaguicidas. Sustancias Químicas Peligrosas. Revista Salud, Trabajo y Ambiente. Consejo Colombiano de Seguridad. IV trimestre. 1998; 5 (18).

26. Urrea E. Situación actual de los neurotóxicos en Colombia. Perpespectivas del siglo XXI. Revista Salud, Trabajo y Ambiente. Consejo Colombiano de Seguridad. III Trimestre de 2000; 7 (25).

27. Administradora de Riesgos Profesionales. Seguro Social. Material Partículado: Sistema de Vigilancia Epidemiológica. Bogotá. 1995

28. Yoshio T. Volatile organic compounds in indoor air. Chemosphere. 1998; 17 (I): 79-82.

29. Universidad del Quindío. Proyección social y formación investigativa del programa de Salud Ocupacional. Facultad Ciencias de la Salud. Armenia (Colombia). 2005.

30. Vasallo de López MI. La Investigación de la Comunicación. Revista Dialogos de la comunicación. Federación Latinoamericana de Facultades de la Comunicación No. 56. Lima. 1999.

31. Eyssautier De La Mora M. Metodología de la Investigación: Desarrollo de la Inteligencia. México. 4a ed. $2002 ;$ p. 217.

32. Brosseau L M, Parker D, Lazovich D, Dugan S, Milton T. Designing intervention effectiveness studies for occupational health and safety: The Minnesota wood dust study. American Journal of Industrial Medicine. 2002; 41:54-61

33. Brosseau L M, Parker D, Lazovich D, Dugan S, Milton T, Pan W. Inhalable dust exposures, tasks, and use of ventilation in small woodworking shops: A pilot Study. Journal Health \& Medical Complete AlHAJ. 2001; 62 (3):322-329.

34. Bullock WH and Laird LT. A pilot study of the particle size distribution of dust in the paper and wood products industry.Association Journal, Health \&Medical Complete. 1994; 55:836

35. Colombia. Ministerio de Protección Social de Colombia. Diagnóstico actual y prospectivo de la salud ocupacional y los riesgos profesionales en Colombia con enfoques de entornos. SENA y El Ministerio. ISBN: 958-97494-7-X. Bogotá; 2005

36. Wonnacott THW and Wonnacott RJW. Introducción a la estadísitca. Limusa Noriega Editores. 2a ed. México. ISBN 968-184509-9. 2008; $\mathrm{p} 784$.

37. Artículos de seguridad S.A. Compendio de Normas legales de Salud Ocupacional en Colombia. ARSEG. Bogotá. 2005

38. Colombia. Ministerio de Protección Social. Resolución 2400 de 1979 Normas sobre vivienda, higiene y seguridad en los establecimientos de trabajo. Ministerio del Trabajo. Bogota. El Ministerio. 1979.

39. Colombia. Ministerio de Protección Social de Colombia. Estadísticas 2003 a junio de 2004. Sistema General de Riesgos Profesionales. Dirección General de Salud Ocupacional y Riesgos Profesionales. Bogotá. El Ministerio. 2004. 